\title{
Leishmaniasis cutánea
}

\author{
T. del Rosal Rabes ${ }^{\mathrm{a}}$, F. Baquero-Artigao ${ }^{\mathrm{b}}$, MJ. García Miguel ${ }^{\mathrm{b}}$ \\ aPediatra. Hospital Infanta Elena. Valdemoro, Madrid. España. \\ bPediatra. Hospital Universitario La Paz. Madrid. España.
}

\begin{abstract}
Resumen
Las leishmaniasis son enfermedades causadas por protozoos del género Leishmania. España es uno de los países en los que la leishmaniasis es endémica. Los casos adquiridos en nuestro país (tanto cutáneos como viscerales) se deben a Leishmania infantum, pero puede haber otros causados por otras especies en viajeros o inmigrantes. Las lesiones cutáneas aparecen fundamentalmente en áreas expuestas y tienden a la curación espontánea dejando cicatriz. El diagnóstico es difícil por la inespecificidad de la clínica y el lento crecimiento del parásito en cultivo. Existen distintas opciones terapéuticas, por lo que cada caso debe ser valorado individualmente según las características de la lesión, la especie causante y el potencial de afectación mucosa. En nuestro país, la primera línea de tratamiento está constituida por los antimoniales pentavalentes intralesionales. En casos refractarios o con riesgo de diseminación mucosa debe emplearse un tratamiento sistémico.
\end{abstract}

Palabras clave: Leishmania. Leishmaniasis cutánea. Tratamiento sistémico. Tratamiento tópico.

\section{Cutaneous leishmaniasis}

\section{Abstract}

Leishmaniasis are a group of diseases caused by protozoa of the genus Leishmania. Spain is one of the countries where leishmaniasis is endemic. All cases acquired in our country (both cutaneous and visceral) are caused by Leishmania infantum, although cases due to other species may appear in travelers or immigrants. Cutaneous lesions appear mainly in exposed areas and tend to heal spontaneously leaving scars. Diagnosis is often difficult due to the non-specific clinical manifestations and the slow growth of the parasite when cultured. There are different available treatment options and each case should be individually considered, taking into account the characteristics of the lesion, the species of Leishmania and the potential for mucosal spread. In Spain, intralesional pentavalent antimonials are the first-line therapeutic option. In those cases at risk for mucosal dissemination or refractory to local therapy, systemic treatment must be given.

Key words: Leishmania. Cutaneous leishmaniasis. Systemic treatment. Topical treatment.

Teresa del Rosal Rabes: teresadelrosal@yahoo.es

Los autores declaran no presentar conflictos de intereses en relación con la preparación y publicación de este artículo. 


\section{Introducción}

La leishmaniasis está constituida por un grupo de enfermedades causadas por varias especies de protozoos del género Leishmania, que son transmitidos a los humanos por dípteros flebotominos hembra (Phlebotomus y Lutzomyia). El reservorio del parásito está formado bien por mamíferos domésticos o salvajes, bien por el ser humano (en regiones endémicas) ${ }^{1}$.

La leishmaniasis puede clasificarse según ${ }^{2,3}$ :

- Las manifestaciones clínicas que produce: lesiones ulcerativas en el lugar de la picadura (leishmaniasis cutánea localizada), múltiples nódulos no ulcerativos (leishmaniasis cutánea difusa), destrucción de las mucosas (leishmaniasis mucosa) e infección visceral diseminada (leishmaniasis visceral).

- La distribución geográfica: Viejo Mundo (Europa, Asia y África) y Nuevo Mundo (Centroamérica y Suramérica). Las especies principales en el Viejo Mundo son $L$. major y L. tropica. En el Nuevo Mundo se distinguen dos subgéneros: L. leishmania (incluye, entre otras, L. mexicana, L. amazonensis y L. chagasi) y L. viannia (incluye L. panamensis, L. brazi- liensis y L. guyanensis). Las especies de Leishmania del Viejo Mundo suelen producir lesiones cutáneas benignas y autolimitadas, mientras que las del Nuevo Mundo pueden causar lesiones graves, incluyendo la afectación mucosa ${ }^{4}$. Las especies del subgénero $L$. viannia se asocian a cuadros más graves y prolongados y presentan el riesgo de desarrollar en el futuro leishmaniasis mucosa, por lo que siempre deben recibir tratamiento sistémico ${ }^{5}$. En el Viejo Mundo los vectores pertenecen al género Phlebotomus y en el Nuevo Mundo, a Lutzomyia.

- La taxonomía (subgénero, complejo y especie).

\section{Epidemiología}

La leishmaniasis cutánea es endémica en más de 70 países del mundo, incluyendo España. Aparece principalmente en trópicos, regiones subtropicales y cuenca mediterránea. Más del $90 \%$ de los casos aparecen en Arabia Saudí, Irán, Afganistán, Brasil y Perúz. Se estima que anualmente se producen 1-1,5 millones de casos, principalmente en personas que viven en áreas endémicas $^{6}$. Sin embargo, también puede afectar a viajeros ${ }^{7,8}$. La incidencia en España 
se desconoce, ya que actualmente no es una enfermedad de declaración obligatoria ${ }^{1}$.

Se han identificado más de 20 especies de Leishmania que pueden originar una patología en humanos. Algunas especies causan enfermedad cutánea, otras visceral y otras ambas 9 . L. infantum es la única especie endémica en España y su principal reservorio es el perro $^{1,3}$. Los casos diagnosticados en nuestro país producidos por otras especies suelen ser consecuencia de viajes a otras zonas endémicas.

\section{Ciclo vital y patogénesis ${ }^{2,10}$}

El parásito presenta dos formas: amastigote (forma redondeada que parasita el sistema mononuclear fagocítico de los mamíferos) y promastigote (forma flagelada que aparece en el vector).

Cuando el mosquito pica a un huésped infectado, ingiere células parasitadas por amastigotes. En el intestino del vector, los amastigotes pasan a promastigotes, se multiplican y migran a la zona bucal. Si el mosquito pica a un ser humano, le inocula promastigotes que entran en las células del sistema mononuclear fagocítico y se convierten en amastigotes. Los amastigotes, parásitos intracelulares obligados, son los que producen las manifestaciones clínicas de la enfermedad en humanos.

\section{Clínica ${ }^{11}$}

La mayoría de infecciones son asintomáticas. El período de incubación va de una semana a varios meses ${ }^{12}$.

\section{Leishmaniasis cutánea}

\section{localizada (botón de Oriente)}

La lesión comienza como una pequeña zona de eritema en el lugar de la picadura que evoluciona a pápula y aumenta de tamaño. Posteriormente, puede ulcerarse en el centro y presentar un borde sobreelevado, bien definido e hiperpigmentado. Las úlceras pueden ser secas o exudativas. En otras ocasiones la lesión no se ulcera, pero puede desarrollar hiperqueratosis o evolucionar a una forma nodular. Son frecuentes las lesiones satélite $^{2,10}$. La mayoría de las veces se ven afectadas las zonas expuestas, no hay clínica sistémica ni dolor local. Pueden aparecer adenopatías regionales ${ }^{5}$. Las lesiones del Viejo Mundo suelen curar espontáneamente en 6-12 meses y dejan cicatriz. La complicación más frecuente es la sobreinfección bacteriana ${ }^{10}$.

\section{Leishmaniasis recidivans}

Se trata de una infección cutánea recurrente poco habitual. Sólo se ha des- 
crito asociada a infecciones por L. tropica. Se considera una reacción de hipersensibilidad crónica en la que aparecen lesiones satélite en los márgenes de lesiones primarias en resolución. Puede durar hasta 20 años.

\section{Leishmaniasis cutánea difusa}

Es una infección diseminada de curso recurrente o crónico, con engrosamiento cutáneo en forma de placas, pápulas y/o nódulos, principalmente en la cara y las extremidades. Las lesiones suelen ser asintomáticas y no presentan tendencia a ulcerarse. Es poco frecuente y se produce por anergia a antígenos de Leishmania. Se observan niveles bajos de IFN- $\gamma$ y TNF- $\alpha^{13}$.

\section{Leishmaniasis mucosa o espundia}

Es una forma casi exclusiva de Suramérica (L. braziliensis) y resulta potencialmente muy grave. Las lesiones mucosas aparecen meses o años después de que las lesiones cutáneas se hayan curado por diseminación hematógena o linfática. Inicialmente se ve afectada la mucosa nasal y se producen la ulceración y la destrucción progresivas del tabique nasal, el paladar, los labios, la faringe y la laringe si no se trata. Nunca se cura espontáneamente ${ }^{3}$.

\section{Diagnóstico $0^{3,1,14}$}

1. Obtención de muestras de piel. Se puede realizar raspado o biopsia de la lesión (del borde de la más reciente y activa) o aspirado (inyectar suero salino estéril y obtener 3-5 muestras de lugares distintos).

2. Técnicas diagnósticas:

- Examen microscópico: tinción con Giemsa para identificar amastigotes.

- Estudio histopatológico de muestras fijadas.

- Cultivo en medio NNN. Se debe mantener al menos 4 semanas, pues el crecimiento puede ser lento, principalmente en casos con baja carga parasitaria.

- Identificación de la especie aislada con anticuerpos monoclonales, análisis de isoenzimas, hibridación con sondas de ácido desoxirribonucleico o reacción en cadena de la polimerasa (PCR). En nuestro medio resulta importante especialmente en viajeros a Centroamérica y Suramérica para descartar una infección por L. braziliensis. La PCR también es muy útil para el diagnóstico en casos con baja carga parasitaria.

La serología no es útil en el diagnóstico de leishmaniasis cutánea, ya que 
es poco sensible y específica, y la mayoría de pacientes no desarrolla una respuesta humoral significativa. Sin embargo, sí puede apoyar el diagnóstico de leishmaniasis mucosa. La intradermorreacción de Montenegro no se emplea actualmente.

\section{Diagnóstico diferencial ${ }^{3,11}$}

En Atención Primaria debe sospecharse leishmaniasis cutánea en lesiones en áreas expuestas que no responden al tratamiento con antibiótico ni corticoides (tabla 1).

\section{Tratamiento}

La leishmaniasis cutánea tiende a curar espontáneamente en varios meses y deja cicatriz. El tratamiento mejora la cicatrización y previene la diseminación parasitaria y las recaídas. Suele indicarse en casos persistentes (duración superior a 6 meses), lesiones múltiples o de gran tamaño (> 4-5 cm), lesiones con repercusión estética o funcional (en la cara o cercanas a las articulaciones) y lesiones causadas por especies de Leishmania con potencial de diseminación mucosa (Nuevo Mundo, subgénero L. viannia). También está indicado en leishmaniasis cutánea difusa y mucosa ${ }^{3,11}$. Cada caso debe valorarse individualmente, por lo que es necesario remitir al paciente a un centro experimentado en el manejo de leishmaniasis cutánea.

No existe ningún tratamiento óptimo. Se han realizado múltiples estudios, pero los datos son difíciles de valorar por la tendencia a la curación espontánea, la inclusión de pocos pacientes, la falta de control con placebo o tratamiento estándar y los cambios en la respuesta a cada fármaco según la especie de Leishmania ${ }^{3,15,16}$. Los antimoniales pentavalentes, parenterales o intralesionales, han sido durante décadas el único

Tabla 1. Diagnóstico diferencial de leishmaniasis cutánea

- Picaduras de insecto

- Úlceras traumáticas

- Nódulos piogénicos

- Granulomas por cuerpo extraño

- Infecciones por micobacterias: tuberculosis cutánea y micobacterias atípicas

- Infecciones fúngicas: paracoccidioidomicosis e histoplasmosis en viajeros a zonas endémicas

- Lepra

- Sarcoidosis

- Esporotricosis

- Sífilis

- Tumores cutáneos 
tratamiento disponible, pero en los últimos años han aparecido nuevas alternativas: amfotericina $B$, pentamidina, miltefosina y termoterapia ${ }^{3}$.

En el Medical Letter se recomienda el tratamiento con antimoniales pentavalentes (estibogluconato sódico o antimoniato de meglumina) por vía parenteral en dosis de $20 \mathrm{mg} / \mathrm{kg} /$ día durante 20 días, o miltefosina oral en dosis de $2,5 \mathrm{mg} / \mathrm{kg} /$ día durante 28 días. Como alternativas aparecen la pentamidina y la paromomicina tópica ${ }^{17}$.

Los antimoniales pentavalentes -estibogluconato sódico (Pentostam ${ }^{\circledR}$ ) y antimoniato de meglumina (Glucanti$\mathrm{me}^{\circledast}$ )- pueden tener efectos adversos graves, aunque habitualmente éstos son reversibles (dolor musculoesquelético, fracaso renal, toxicidad hepática y cardíaca) cuando se administran por vía parenteral. Para intentar disminuirlos se han propuesto pautas de tratamiento más cortas (10 días) en casos sin riesgo de diseminación mucosa ${ }^{3,18}$. En los últimos años han aumentado los casos de fracaso terapéutico por resistencia parasitaria a los fármacos ${ }^{19} \mathrm{o}$ por inmunosupresión del paciente ${ }^{3}$.

La administración intralesional de antimoniales pentavalentes puede ser muy efectiva y tiene muchas ventajas: se alcanza una alta concentración del fármaco en el lugar de la infección y se reducen la toxicidad sistémica y los costes derivados del tratamiento. Es el tratamiento de elección de la leishmaniasis cutánea localizada en nuestro medio, pero debe ser realizado por personal con experiencia ${ }^{4}$. Se administran 0,2-1 $\mathrm{ml}$ inyectándolos en varios puntos de la lesión y se repite la administración cada 1-3 semanas, con un número de dosis variable según la evolución. Sólo puede emplearse en leishmaniasis cutánea del Viejo Mundo y en infecciones por $L$. mexicana cuando las lesiones son únicas y pequeñas. En el resto de especies del Nuevo Mundo o cuando las lesiones son múltiples o de gran tamaño (mayores de $5 \mathrm{~cm}$ ), hay diseminación metastásica o fracasa el tratamiento local, debe emplearse un tratamiento parenteral ${ }^{1,4,20}$.

La pentamidina se asocia frecuentemente con efectos secundarios (náuseas, anorexia, mareo, prurito, hipotensión, necrosis en el lugar de punción, alteraciones hematológicas y electrolíticas), principalmente cuando se administran dosis elevadas. Por este motivo se reserva para casos refractarios o cuando no hay otros tratamientos disponibles. Al igual que ocurre con los antimoniales, están aumentando los casos resistentes ${ }^{21}$. 
La paromomicina tópica (Leshcutan ${ }^{\circledR}$ ) no está disponible en España ni en Estados Unidos y debe reservarse para los casos sin riesgo de afectación mucosa ${ }^{17}$.

La miltefosina sólo ha sido ensayada en pacientes de más de 12 años y demuestra una efectividad variable según la especie de Leishmania. Sus efectos secundarios más frecuentes son náuseas, mareo, dolor de cabeza y aumento de creatinina. Su principal ventaja es la administración oral22.

Los azoles orales (fluconazol, itraconazol) son una alternativa en lesiones complejas o con potencial de afectación mucosa. Se toleran muy bien pero sólo son efectivos frente a algunas especies $^{4,5,23}$.

La amfotericina B deoxicolato es activa frente a Leishmania, pero los efectos adversos son frecuentes (hiperpirexia, malestar general, hipotensión, tromboflebitis, daño renal, hipopotasemia, anemia y hepatitis). Las formulaciones lipídicas de amfotericina $B$ son mucho menos tóxicas y han demostrado eficacia en el tratamiento de leishmaniasis visceral ${ }^{4}$. Su coste ha limitado su uso en leishmaniasis cutánea. Existen pocos estudios, pero parece que podrían ser útiles tanto por vía tópica como por vía parenteral con escasos efectos secunda$\operatorname{rios}^{24-28}$.

Otros posibles tratamientos que se han empleado son el imiquimod tópico (habitualmente en asociación con otros tratamientos), la termoterapia y la crioterapia ${ }^{20,29-32}$. No se recomienda la escisión quirúrgica por el alto riesgo de recaída local y desfiguración, con la excepción de la biopsia-extirpación de lesiones pequeñas que no hayan podido diagnosticarse con el frotis ${ }^{1,9}$.

\section{Prevención ${ }^{10}$}

En la actualidad no existe una vacuna disponible, por lo que la prevención se basa en las medidas frente a picaduras de mosquitos en viajeros y el control de reservorios animales.

\section{Bibliografía}

1. García-Almagro D. Leishmaniasis cutánea. Actas Dermosifiliogr. 2005;96:1-24.

2. Leder K, Weller PF. Epidemiology and clinical manifestations of leishmaniasis. UpToDate,
2008 [actualizado el 11/03/2008; consultado el 20/12/2009]. Disponible en www.uptodate. com

3. Reithinger $\mathrm{R}$, Dujardin JC, Louzir $\mathrm{H}_{\text {, Pirmez }}$ C, Alexander B, Brooker S. Cutaneous leishmaniasis. Lancet Infect Dis. 2007;7:581-96. 
4. Blum J, Desjeux P, Schwartz E, Beck B, Hatz C. Treatment of cutaneous leishmaniasis among travellers. J Antimicrob Chemother. 2004;53:158-66.

5. Bailey MS, Lockwood DN. Cutaneous leishmaniasis. Clin Dermatol. 2007;25:203-11.

6. Desjeux P. Focus: Leishmaniasis. Nat Rev Microbiol. 2004;2:692-3.

7. Lawn SD, Whetham J, Chiodini PL, Kanagalingam J, Watson J, Behrens $\mathrm{RH}$, et al. New world mucosal and cutaneous leishmaniasis: an emerging health problem among British travellers. QJM. 2004;97:781-8.

8. Scope A, Trau H, Anders G, Barzilai A, Confino $Y$, Schwartz E. Experience with New World cutaneous leishmaniasis in travelers. J Am Acad Dermatol. 2003;49:672-8.

9. Markle WH, Makhoul K. Cutaneous leishmaniasis: recognition and treatment. Am Fam Physician. 2004;69:1455-60.

10. Ortega-Barria E, Romero LI. Leishmania Species (Leishmaniasis). En: Long SS, editor. Principles and Practice of Pediatric Infectious Diseases. 3. ${ }^{a}$ ed. Philadelphia: Churchill-Livingstone; 2008. p. 1246-53.

11. David CV, Craft N. Cutaneous and mucocutaneous leishmaniasis. Dermatol Ther. 2009; 22:491-502.

12. Melby PC. Experimental leishmaniasis in humans: review. Rev Infect Dis. 1991;13:1009-17.

13. Turetz ML, Machado PR, Ko Al. Disseminated leishmaniasis: a new and emerging form of leishmaniasis observed in northeastern Brazil. J Infect Dis. 2002;186:1829-34.

14. Leder K, Weller PF. Diagnosis of leishmaniasis. UpToDate, 2007 [actualizado el 08/06/ 2007; consultado el 20/12/2009]. Disponible en www.uptodate.com

15. Minodier P, Parola P. Cutaneous leishmaniasis treatment. Travel Med Infect Dis. 2007;5: 150-8.
16. González U, Pinart M, Reveiz L, Alvar J. Interventions for Old World cutaneous leishmaniasis. Cochrane Database Syst Rev. 2008: CD005067.

17. Drugs for parasitic infections. Treat Guidel Med Lett. 2007;5:e1-e15.

18. Wortmann G, Miller RS, Oster C, Jackson J, Aronson N. A randomized, double-blind study of the efficacy of a 10- or 20-day course of sodium stibogluconate for treatment of cutaneous leishmaniasis in United States military personnel. Clin Infect Dis. 2002;35:261-7.

19. Croft SL, Sundar S, Fairlamb AH. Drug resistance in leishmaniasis. Clin Microbiol Rev. 2006;19:111-26.

20. Khatami A, Firooz A, Gorouhi F, Dowlati $Y$. Treatment of acute Old World cutaneous leishmaniasis: a systematic review of the randomized controlled trials. J Am Acad Dermatol. 2007;57(2):335.e1-29.

21. Leder K, Weller PF. Treatment and prevention of leishmaniasis. UpToDate, 2009 [actualizado el 02/10/2009; consultado el 20/12/ 2009]. Disponible en www.uptodate.com

22. Soto J, Toledo JT. Oral miltefosine to treat new world cutaneous leishmaniasis. Lancet Infect Dis. 2007;7:7.

23. Alrajhi AA, Ibrahim EA, De Vol EB, Khairat $M$, Faris RM, Maguire $\mathrm{JH}$. Fluconazole for the treatment of cutaneous leishmaniasis caused by Leishmania major. N Engl J Med. 2002;346:891-5.

24. Brown $M$, Noursadeghi $M$, Boyle J, Davidson RN. Successful liposomal amphotericin B treatment of Leishmania braziliensis cutaneous leishmaniasis. Br J Dermatol. 2005;153:203-5.

25. Torre-Cisneros J, Prada JL, Villanueva JL, Valverde F, Sánchez-Guijo P. Successful treatment of antimony-resistant cutaneous leishmaniasis with liposomal amphotericin B. Clin Infect Dis. 1994;18:1024-5. 
26. Zvulunov A, Cagnano E, Frankenburg $S$, Barenholz Y, Vardy D. Topical treatment of persistent cutaneous leishmaniasis with ethanolic lipid amphotericin B. Pediatr Infect Dis J. 2003;22: 567-9.

27. Amato V, Rabello A, Rotondo-Silva A. Successful treatment of cutaneous leishmaniasis with lipid formulations of amphotericin B in two immunocompromised patients. Acta Trop. 2004; 92:127-32.

28. Del Rosal T, Baquero-Artigao F, García Miguel MJ, De Lucas R, Del Castillo F. Successful Treatment of Childhood Cutaneous Leishmaniasis with Liposomal Amphotericin B: Report of Two Cases. J Trop Pediatr. 2010;56: 122-4.

29. Arévalo I, Tulliano G, Quispe A. Role of imiquimod and parenteral meglumine antimoniate in the initial treatment of cutaneous leishmaniasis. Clin Infect Dis. 2007;44:154954.

30. Reithinger $R$, Mohsen $M$, Wahid M. Efficacy of thermotherapy to treat cutaneous leishmaniasis caused by Leishmania tropica in Kabul, Afghanistan: a randomized, controlled trial. Clin Infect Dis. 2005;40:1148-55.

31. Vega JC, Sánchez BF, Montero LM. The efficacy of thermotherapy to treat cutaneous leishmaniasis in Colombia: a comparative observational study in an operational setting. Trans $R$ Soc Trop Med Hyg. 2009;103:703-6.

32. Asilian A, Sadeghinia A, Faghihi G, Momeni $A$. Comparative study of the efficacy of combined cryotherapy and intralesional meglumine antimoniate (Glucantime) vs. cryotherapy and intralesional meglumine antimoniate (Glucantime) alone for the treatment of cutaneous leishmaniasis. Int J Dermatol. 2004;43:281-3. 